\title{
Abdominal Splenosis Mimicking a Colon Tumour
}

\author{
Joana Braga, Francisca Pereira, Cristiana Fernandes, Marinha Silva, Teresa Boncoraglio, Carlos Oliveira \\ Department of Internal Medicine, Hospital Santa Maria Maior, Barcelos, Portugal
}

Doi: 10.12890/2021_002219 - European Journal of Case Reports in Internal Medicine - ๑ EFIM 2021

Received: $13 / 12 / 2020$

Accepted: $23 / 12 / 2020$

Published: $18 / 01 / 2021$

How to cite this article: Braga J, Pereira F, Fernandes C, Silva M, Boncoraglio T, Oliveira C. Abdominal splenosis mimicking a colon tumour. EJCRIM 2021;8: doi:10.12890/2020_002219.

Conflicts of Interests: The Authors declare that there are no competing interests.

This article is licensed under a Commons Attribution Non-Commercial 4.0 License

\section{ABSTRACT}

Splenosis is a benign condition which results from the self-implantation of splenic tissue on intra or extraperitoneal surfaces, after splenic trauma or splenectomy. Patients are usually asymptomatic but may present with varied symptoms related to the implantation site. The diagnosis is a challenge because abdominal splenosis can mimic several diseases, including neoplasm. The gold standard examination for its diagnosis is scintigraphy with $99 \mathrm{mTc}$-labelled heat-denatured erythrocyte. When splenosis is found in an asymptomatic patient, surgical removal is not indicated. A 57-year-old male patient presented with sporadic epigastric pain and a suspected mass in the recto-sigmoid transition. Abdominal ultrasound, CT and MRI identified this mass, its characteristics and location, but failed to distinguish its nature. However, given the patient's past history of splenectomy and because the mass showed a similar sign to that of the splenic parenchyma, a hypothesis of abdominal splenosis was raised, which was confirmed by scintigraphy with $99 \mathrm{mTc}$-labelled heat-denatured erythrocyte.

In this case, the diagnosis was obtained before the patient was subjected to more invasive procedures, which are associated with high morbidity, and, as in most cases, no targeted intervention was necessary.

\section{LEARNING POINTS}

- Increasing numbers of cases of abdominal trauma will result in more frequent splenosis.

- Diagnosis is sometimes complex as splenosis mimics several diseases.

- The usual complementary imaging studies often fail to diagnose this entity so clinical suspicion is fundamental for correct diagnosis and treatment.

\section{KEYWORDS}

Splenosis, abdominal trauma, scintigraphy, fragile erythrocytes

\section{CASE DESCRIPTION}

A 57-year-old male patient was referred for an oncology consultation for further study of a suspected mass noted on digital rectal examination and later documented on abdominal ultrasound and abomino-pelvic CT. The mass was located between the bladder and rectalsigmoid junction wall with infiltration of the perirectal fat (Fig. 1).

Acomplete anamnesis and physical examination were performed. The patient had dyslipidaemia, type 2 diabetes, a right total hip replacement, left iliofemoral deep vein thrombosis, benign prostatic hyperplasia and a splenectomy at 21 years of age after a car accident. The patient had experienced sporadic epigastric pain for a few months, without relief or aggravating factors, and unrelated to food intake. He denied nausea, vomiting, weight loss, rectal haemorrhage, melena, changes in intestinal transit or other symptoms. On digital rectal examination, a mass was palpable $6 \mathrm{~cm}$ from the anal margin, and was painless to palpation. The remaining physical examination and analytical study were unremarkable. 
To complement the aetiological study, the patient underwent a colonoscopy that showed no changes and pelvic magnetic resonance imaging (MRI) that demonstrated a large ovoid mass $(7 \times 5 \times 3 \mathrm{~cm})$ in the rectovesical pouch, contacting the anterior wall of the rectum and posterior to the bladder, with an identical sign to that of the normal splenic parenchyma (Fig. 2). In light of the patient's medical history and the MRI findings, it was decided to perform scintigraphy. Biopsy of the mass was postponed until the scintigraphy results were received.

Scintigraphy revealed several foci of anomalous accumulation of fragile erythrocytes, on the left flank, in the transition from the flank to the right iliac fossa and a large focus on the hypogastric projection, apparently located posterior to the bladder (the location of the mass under study) compatible with splenosis (Fig. 3). A biopsy was therefore not necessary.

As the patient was asymptomatic, he did not need any targeted treatment.

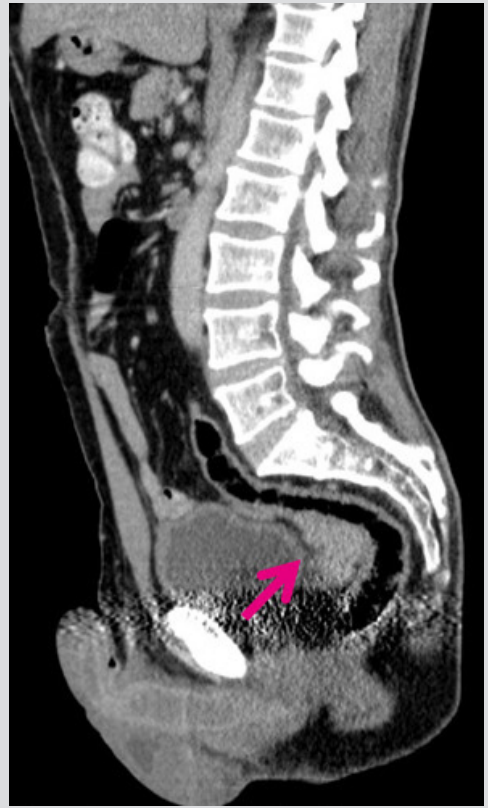

Figure 1. Arrow identifying the mass between the bladder and the recto-sigmoid junction

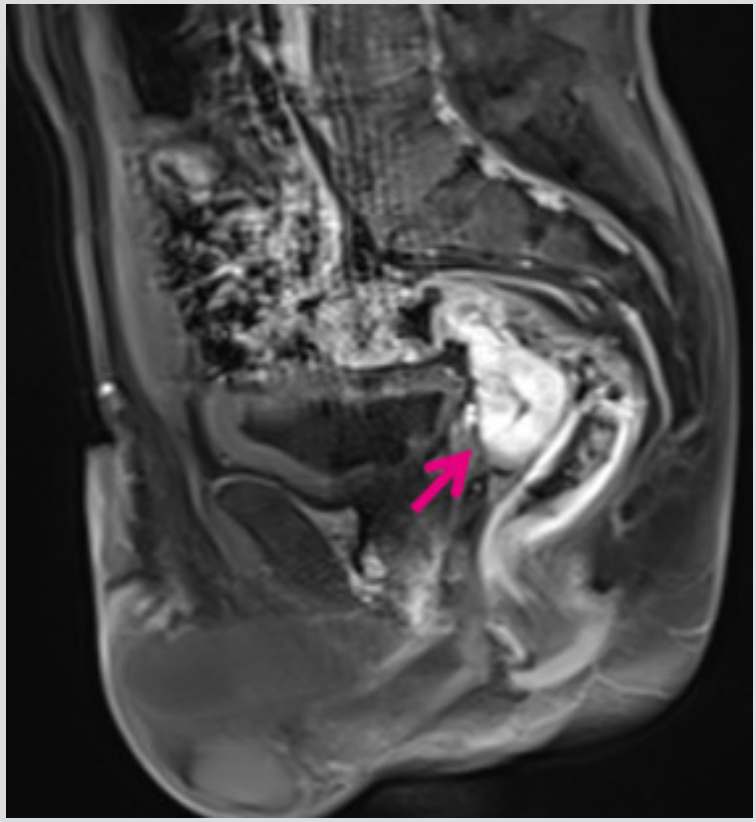

Figure 2. Arrow identifying a large ovoid mass in the rectovesical pouch

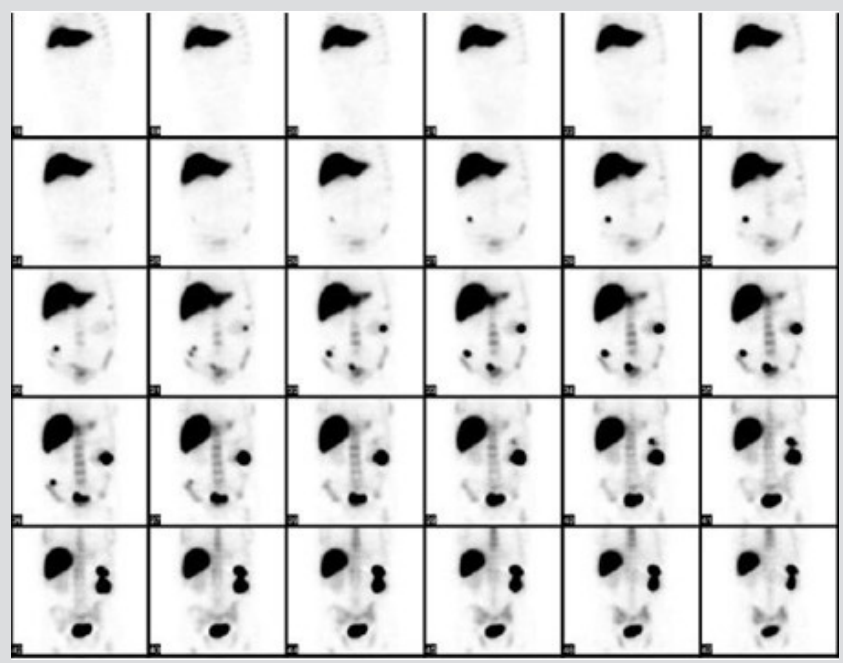

Figure 3. Scintigraphy showing several foci of anomalous accumulation of fragile erythrocytes, compatible with splenosis

\section{DISCUSSION}

Splenosis is an acquired and benign condition, which results from the self-implantation of one or more deposits of splenic tissue on intra or extraperitoneal surfaces, after splenic trauma or splenectomy ${ }^{[1,2]}$. Its true incidence is unknown, since its diagnosis is usually an incidental finding ${ }^{[1]}$. 
Patients are usually asymptomatic but may present with varied symptoms depending on the location of the ectopic splenic tissue [2, 3]. The most frequent locations are the peritoneum, the omentum and the mesentery, but other locations such as the pericardium, subcutaneous tissue and even brain tissue have also been described ${ }^{[1,4]}$.

The implanted tissue is able to re-establish circulation and grow. The dimensions can vary from a few millimetres to more significant dimensions (up to $12 \mathrm{~cm}$ ), however, the diameter is usually less than $3 \mathrm{~cm}$ given the limited blood supply ${ }^{[2]}$. Most cases of splenosis result from urgent splenectomy due to abdominal trauma, generally at a young age. The time interval between trauma and the appearance of abdominal splenosis varies from 5 months to 32 years, with an average of 10 years, which reflects its slow growth ${ }^{[2,3]}$. The diagnosis can be a challenge because splenosis can mimic several diseases, including neoplasm ${ }^{[3-5]}$. Thus, a detailed clinical history, personal history (namely abdominal trauma), objective examination and adequate imaging exams are essential in order to obtain a correct diagnosis, thus preventing the patient from being subjected to more invasive procedures (such as biopsy or laparotomy) that are unnecessary and associated with high morbidity. A preoperative diagnosis requires a high level of suspicion and should be established with caution. There is a wide variety of imaging exams, such as ultrasound, computed tomography and MRI, which have limited value in the diagnosis of splenosis as demonstrated in this case. These modalities are able to visualize these lesions, assess their shape and dimensions, but not their exact nature ${ }^{[4]}$. The gold standard exam for diagnosis is scintigraphy ${ }^{[1,3,6]}$. When splenosis is a finding in an asymptomatic patient, surgical removal is not indicated. However, the surgical approach is recommended in patients whose diagnosis is uncertain, especially when scintigraphy is not readily available and there is a suspicion of neoplastic disease, as well as in patients with haematological disease recurrence ${ }^{[2]}$.

As described in this case, splenosis is a relatively benign condition, but it mimics more serious pathologies, namely neoplasms that require a more urgent approach. Thus, splenosis should be part of the differential diagnosis in patients with suspected abdominal masses and a history of abdominal trauma or splenectomy ${ }^{[5]}$. Hopefully, we can prevent the patient from being subjected to unnecessary invasive interventions.

\section{REFERENCES}

1. Gupta K, Ahluwalia A, Jain T, Saggar K. Abdominal splenosis mimicking peritoneal deposits- a case report. Pan Afr Med J 2014;17:1-4.

2. Moreira R, Paula I, Silva M, Bernardes B, Capanema H. Relato de caso de esplenose: diagnóstico diferencial importante em pacientes esplenectomizados apresentando massas abdominais. Rev da Univ Val do Rio Verde 2014;12(1):748-754.

3. Ksiadzyna D, Peña AS. Abdominal splenosis. Rev Española Enfermedades Dig 2011;103(8):421-426.

4. Ribeiro JC, Silva CM, Santos AR. Splenosis. A diagnosis to be considered. Int Braz J Urol 2006;32(6):678-680.

5. Liu Y, Ji B, Wang G, Wang Y. Abdominal multiple splenosis mimicking liver and colon tumors: a case report and review of the literature. Int J Med Sci 2012;9(2):174-177.

6. Bidet AC, Dreyfus-Schmidt G, Mas J, Combe J, Milleret P, Bidet R. Diagnosis of splenosis: the advantages of splenic scintiscanning with Tc $99 \mathrm{~m}$ heat-damaged red blood cells. Eur J Nucl Med 1986: 12(7):357-358. doi: 10.1007/BF00263820. 